\title{
Factores que inciden en el consumo de tabaco en los estudiantes de ingeniería: Estudio piloto
}

\section{Factors affecting tobacco use in engineering students: pilot study}

Gloria Elizabeth Gavino Díaz. ', Erika Jazmín López Rivadeneira. ${ }^{2}$, Víctor Hugo Guaraca Parreño. ${ }^{3}$ \& Holguer Estuardo Romero Urrea. ${ }^{4}$

Recibido: 13-07-2019 / Revisado: 19-08-2019 /Aceptado: 07-09-2019/ Publicado: 04-10-2019

\begin{abstract}
.
DOI: https://doi.org/10.33262/cienciadigital.v3i4.910

Smoking is an addiction that generates a respiratory condition that gradually impairs lung function, manifesting symptomatically with several signs that the individual may perceive and that make development impossible normal functions; constituted not only a serious clinical situation, but a public health problem. This warranted a decisive study of those influential factors that generated a pattern of consumerism in the individuals studied and which in turn establish common consequences related to this addiction. Objectives: To determine the factors associated with smoking in students of the Faculty of Engineering; establish the incidence; identify the correlation between psychological and social factors in tobacco use. Materials and methods: The study of mixed, transversal, descriptive and co-relational type, phenomenological; are used as instruments: a test validated by expert judgement and an in-depth interview based on open questions related to the categories associated with smoking: psychological, social and family where the problem is sought to analyze the problem to reach etiopathogenic factor and thus reach the consequences and come up with solutions. Population: 153 students. Results: As a result of the pilot test, $77.8 \%$ of the male population aged 22 to 24 years who are smokers were found, 55.6\% said they smoke occasionally. $66.67 \%$ of men aged 22 to 24 consumed 1 to 3 cigarettes and $11.11 \%$ consumed 4 to 6 cigarettes; $44.4 \%$ of male-born youth between the ages of 22 and 24 consume cigarettes
\end{abstract}

\footnotetext{
${ }^{1}$ Universidad Estatal de Milagro, Facultad Ciencias de la Salud, Ecuador, ggavinod@unemi.edu.ec

${ }^{2}$ Universidad Estatal de Milagro, Facultad Ciencias de la Salud, Ecuador, erijazlr12@gmail.com

${ }^{3}$ Universidad Estatal de Milagro, Facultad Ciencias de la Salud, Ecuador, mdvictorhgp@hotmail.com

${ }^{4}$ Universidad Estatal de Milagro, Facultad Ciencias de la Salud, Ecuador, hromerou@unemi.edu.ec
} 
when they feel uneasy; $55.56 \%$ of young people use tobacco in the company of their friends. Conclusions: Smoking is determined as a very common addiction in young adults on which this study has been based. The reliability of the results could be validated to establish an effective incidence range.

Keywords: Tobacco, Addiction, Smoking, Cigarette, Consumption.

\section{Resumen.}

El tabaquismo es una adicción que genera una afección de tipo respiratorio que deteriora la función pulmonar paulatinamente, manifestándose sintomatológicamente con varias señales que el individuo puede percibir y que imposibilitan el desarrollo normal de sus funciones; constituyéndose esto no solo en una situación clínica grave, sino en un problema de salud pública. Esto ameritó un estudio determinante de aquellos factores influyentes que generaron un patrón de consumismo en los individuos estudiados y que a su vez establecen consecuencias comunes relacionadas con esta adicción. Objetivos: Determinar los factores asociados al tabaquismo en los estudiantes de la Facultad de Ingeniería; establecer la incidencia; identificar la correlación entre los factores psicológicos y sociales en el consumo de tabaco. Materiales y métodos: El estudio de tipo mixto, transversal, descriptivo y correlacional, fenomenológica; se usan como instrumentos: un test validado por juicio de expertos y una entrevista a profundidad elaborada a base de preguntas abiertas relacionadas a las categorías asociadas al tabaquismo: psicológica, social y familiar donde se busca analizar el problema para llegar al factor etiopatogénico y de esta manera llegar a las consecuencias y plantear soluciones. Población: 153 estudiantes. Resultados: Como resultado en base al test piloto se encontró un $77,8 \%$ de población masculina con edades entre 22 a 24 años que son fumadores, 55,6\% afirmó que fuman ocasionalmente. El 66,67\% de hombres de 22 a 24 años consume de 1 a 3 cigarrillos y $11,11 \%$ consume de 4 a 6 cigarrillos; $44,4 \%$ de los jóvenes de género masculino entre 22 a 24 años consume cigarrillos cuando sienten intranquilidad; el 55,56\% de jóvenes consume tabaco en compañía de sus amistades. Conclusiones: El tabaquismo se determina como una adicción muy común en los adultos jóvenes. Se pudo validar la confiabilidad de los resultados para establecer un rango de incidencia efectivo.

Palabras claves: Tabaco, Adicción, Tabaquismo, Cigarrillo, Consumo.

\section{Introducción.}

El consumo de tabaco es una adicción que provoca afecciones de tipo respiratorio y se constituyen en el problema más común dentro del ámbito de la salud pública a nivel mundial. Esta problemática conlleva varios factores que están relacionados y que pueden originar una 
dependencia a la sustancia adictiva. Fumar está asociado con diversos factores, los cuales se planteó como de tipo psicológico: autoestima, estrés, ansiedad, temores, influencia de la publicidad; de tipo social: familia, amigos, compañeros de trabajo y pareja. Se dice que, en los años de transición entre la enseñanza media y superior, más usuarios del tabaco inician, desarrollan y estabilizan su comportamiento de fumar. (Carrión, D. 2017). Los principales determinantes y condicionantes del tabaquismo en la adolescencia son factores socioeconómicos, de comportamiento y personales. A pesar de que las investigaciones sobre adolescentes son escasas en Ecuador, se ha visto que el uso inicial de tabaco es bastante prematuro en la vida estudiantil. El Instituto Nacional de Cáncer identificó que el inicio del hábito tabáquico ocurre, en promedio, entre los 12 y 13 años, etapa que coincide con la transición de la infancia a la vida adulta, fase en la que acontecen una serie de transformaciones fisiológicas, comportamentales y psicosociales. (Armijos Ordóñez, L. E. 2017). Trastornos mentales como depresión, soledad y otros síntomas tienden a un mayor consumo de sustancias psicoactivas durante la adolescencia y posteriormente durante la vida adulta. (Zander Neves, C. 2018). Además de estos factores, se estableció un análisis directo de daños biológicos que suelen aparecer a consecuencia del consumo de tabaco entre los cuales están: daños respiratorios y cardiovasculares, problemas gástricos, afecciones de la piel, afecciones dentales, enfermedades óseas y problemas de salud reproductiva. En vista de que la mayoría de los factores mencionados pueden verse combinados e interactuando entre sí, se los definió como parte primordial de la investigación; lo cual se confirmó al aplicar la metodología y observar las diferentes reacciones de los individuos y establecer los cuestionamientos relacionados con la problemática.

La Organización Mundial de la Salud (OMS) estima que la mortalidad atribuida específicamente al tabaco es de $12 \%$ a nivel mundial y de $16 \%$ en las Américas (17\% en los hombres y $15 \%$ en las mujeres) (Monteiro, M. G. 2007). De las defunciones asociadas con las enfermedades no transmisibles, el tabaco es responsable de $15 \%$ de las muertes por enfermedades cardiovasculares, $26 \%$ de las muertes por cáncer y $51 \%$ de las muertes por enfermedades crónicas respiratorias (Martínez, J. C. 2016). El rol nocivo del tabaco ha sido considerablemente demostrado desde hace mucho tiempo, compone uno de los problemas más graves de salud pública a nivel mundial y se ha determinado como la gran epidemia silenciosa del siglo XX. Surgió claramente asociado al progreso y la industrialización, y es la raíz de tres millones de muertes al año, con una tendencia progresiva. Se estima que, si las prácticas de consumo no varían, en el año 2020 la mortalidad atribuible al tabaco superaría los ocho millones de muertes al año (Wünsch Filho, V. 2010). El consumo de tabaco es uno de los principales factores de riesgo de varias enfermedades crónicas, como el cáncer y las enfermedades pulmonares y cardiovasculares, en su informe publicado del año 2017 se estimó que 2700 millones de personas siguen sin protección contra las enfermedades, la discapacidad y la muerte que provocan el tabaquismo y la exposición al humo ajeno, o contra los daños económicos, ambientales y sociales conexos. Por un lado, el consumo del producto y la exposición a su humo causan una amplia gama de enfermedades, con devastadoras 
consecuencias no solo sanitarias, sino también sociales, económicas y ambientales. Por otro, muchas de las estrategias y medidas para combatir esta verdadera epidemia mundial, que cuentan con una sólida evidencia científica, han sido ya probadas en países de diferentes contextos sociales y económicos y no requieren de altos presupuestos para su ejecución. (Danel, I. 2018) (Organización Mundial de la Salud, 2017).

El estudio de esta problemática es valioso desde el punto de vista de la salud pública, ya que las estadísticas se extienden a factores resaltantes que afectan este tipo de población, como se muestra en un estudio realizado en México, cuyo propósito se basó en evaluar la dependencia física y psicológica de los fumadores mexicanos y su asociación con factores físicos, psicológicos y sociales. Esto se realizó a través de las escalas FNDT y TAPDS de la Encuesta Nacional de consumo de drogas, alcohol y tabaco (ENDOCAT). Los resultados que se obtuvieron fueron de $82.3 \%$ de fumadores diarios y $98.8 \%$ de ocasionales que reportaron dependencia física leve, mientras que 47.9 y $37.9 \%$, respectivamente, presentaron dependencia psicológica moderada (Paz-Ballesteros, W. 2019). La edad de inicio temprana de consumo de tabaco, uso de drogas, consumo alto de alcohol y malestar emocional se asociaron con niveles altos de dependencia psicológica en todos los fumadores. El grupo de fumadores jóvenes varones de entre 15-34 años se caracteriza por acceder fácilmente al tabaco, tener una menor percepción del daño ocasionado por este y no estar motivados para realizar un intento de abandono (Castedo, C. R. 2019). Algunos estudios realizados sobre los estilos de vida de los estudiantes universitarios han demostrado la existencia de estilos de vida poco saludables, sugiriéndose además que, durante la etapa universitaria, los estudiantes abandonan hábitos saludables y adquieren otros nocivos (Cecilia, M. J. 2019). No hay duda de que ciertos tipos de conductas como el tabaquismo, entre otros podrían contribuir por sí solos a aumentar la morbilidad e, incluso, la mortalidad. De manera general se establece el hábito del tabaquismo como una adicción generada por el contenido de nicotina del cigarrillo, ocasionando esto una posición de dependencia que puede conllevar muchas veces al consumo de otras drogas más adictivas; y su incidencia aumenta más día con día. Si bien no todos los fumadores son adictos, la mayoría de los fumadores diarios sí lo son, en los cuales es de la máxima importancia que dejen de fumar, ya que son los más expuestos a los graves daños que produce el consumo de tabaco. El concepto de dependencia es muy importante para los médicos clínicos, ya que las manifestaciones clínicas dependen de las características individuales de personalidad de cada individuo, así como de las circunstancias socioculturales que lo rodean. El principal síntoma, en los fumadores dependientes, es la necesidad imperiosa o compulsiva de volver a consumir la sustancia para experimentar la recompensa que produce y para evitar el síndrome de abstinencia (Bello, S. 2009). En México, la Encuesta Nacional de Consumo de Drogas, Alcohol y Tabaco (ENCODAT, 2016- 2017), realizada en población de 12 a 65 años, indica que existen 14.9 millones (17.6\%) de fumadores activos (11 millones 78 mil hombres y 3 millones 812 mil mujeres); 6.4\% señaló fumar diariamente un promedio de 7.4 cigarros; la edad de inicio de consumo diario es de 21.4 años, en las mujeres, y de 18.8 años, en los hombres (Mandujano, J. L. 2018). 
El propósito que enmarca la determinación de los factores asociados al consumo de tabaco, radica en que, desde el punto de vista de la salud pública tanto a nivel global como a nivel local se establece el hábito del tabaquismo como una adicción generada por el contenido de nicotina del producto (cigarrillos), un factor de riesgo común para seis de las ocho principales causas de muerte, responsables (estas ocho) de casi dos tercios del total de defunciones a nivel mundial, que en contribución esto ocasiona una posición de dependencia que puede conllevar muchas veces al consumo de otras drogas más adictivas; y su incidencia aumenta más día con día. Se ha concretado investigar los factores asociados al consumo de tabaco a un porcentaje de estudiantes universitarios de la localidad, correspondiente a la Facultad de Ingeniería de la Universidad Estatal de Milagro; logrando de esta manera exponer como éstos se relacionan e influencian a los individuos de forma directa o indirecta generando la dependencia al adictivo, así mismo esto permitirá establecer un nivel de incidencia actual que servirá como base para plantear soluciones; además se resalta la relevancia del estudio ya que la problemática envuelve varios aspectos sociales y psicológicos que deben tenerse en cuenta al momento de plantear el por qué la problemática ha aumentado de manera rápida las últimas décadas, afectando no solo a individuos en etapa adulta sino a población más joven e incluso desde la niñez. Todo esto también relacionando los daños biológicos más frecuentes en consecuencia a esta adicción.

Los datos requeridos para el estudio se determinaron mediante una metodología de tipo mixta, aplicando un cuestionario en base a la determinación de factores psicológicos y sociales (Hernández Sampieri, R. 2016), así como de los daños biológicos que esta adicción conlleva. Se dice que el estrés es un factor que se ha relacionado con el inicio y el mantenimiento del consumo de tabaco; es importante conocer dicho aspecto, pues algunos autores señalan que el ingreso a la universidad genera estrés por la necesidad de adaptarse a un nuevo ambiente y a nuevas demandas. También se identifican síntomas de depresión y ansiedad, así como un alto afecto negativo. Se sabe que la comorbilidad de ansiedad y depresión es un factor que aumenta la probabilidad del consumo de sustancias, particularmente en el caso del consumo de tabaco (Mandujano, J. L. 2018). Por lo tanto, identificar las situaciones que generan estrés podría ayudar a determinarlo como uno de los factores asociados al consumo de tabaco ya que, al ser el tabaquismo una enfermedad adictiva, se hace necesario, en cada fumador, caracterizar y objetivar la dependencia y su severidad, tanto en sus aspectos físicos como psicosociales.

\section{Metodologia.}

La metodología que se utilizó en el presente estudio tiene un enfoque mixto (cualicuantitativo), es de tipo transversal, descriptivo y correlacional, en el cual se especificó las características y perfil de la población, y se determinó fenomenológicamente las causales del problema a profundidad. Por otro lado, como el objetivo lo plantea se buscó conocer la relación entre los factores investigados de manera particular y vincular su efecto relativo al 
consumo de cigarrillo. Según su contexto se determinó como una investigación de campo, en la cual se aplicó la técnica de observación durante la ejecución del test validado. El instrumento mencionado es un test debidamente certificado por expertos del área de la salud y especialistas. En el instrumento de evaluación se detallan 16 preguntas formuladas acorde a los objetivos planteados inicialmente: Los ítems 4, y 5 del cuestionario están asociados al objetivo general de la investigación que es determinar los factores asociados al consumo de tabaco. Los ítems 1, 2 y 3 están en relación al objetivo específico 1 que es establecer la incidencia del tabaquismo en los estudiantes de la facultad. De los ítems 7 al 12 se busca cumplir con el objetivo específico 2 que es identificar los factores psicológicos y sociales asociados al consumo de tabaco. Los ítems 6, y del 13 al 16 están relacionados al objetivo específico 3 que guarda relación con los daños asociados al tabaquismo; a continuación, se muestran las preguntas utilizadas en el cuestionario:

Tabla 1. Ítems utilizados en el cuestionario "Factores asociados al consumo de tabaco"

\begin{tabular}{|c|c|c|c|}
\hline 1 & $\begin{array}{l}\text { ¿Usted fuma? } \\
\text { Si, No }\end{array}$ & 9 & $\begin{array}{l}\text { ¿Le dan deseos de fumar cuando se siente } \\
\text { estresado? } \\
\mathrm{Si}, \mathrm{No}\end{array}$ \\
\hline 2 & $\begin{array}{|lr|}\text { ¿Con que frecuencia fuma usted? } \\
\text { Mensualmente, } \\
\begin{array}{lr}\text { Diariamente, } \\
\text { ninguna }\end{array} & \text { Semanalmente, } \\
\end{array}$ & 10 & $\begin{array}{l}\text { ¿Se siente fácilmente influenciado por la } \\
\text { publicidad del cigarrillo? } \\
\mathrm{Si}, \mathrm{No}\end{array}$ \\
\hline 3 & $\begin{array}{l}\text { ¿Cuándo usted fuma, ¿cuántos cigarrillos } \\
\text { consume? } \\
\text { Más de } 1 \text { caja de cigarrillos, De } 7 \text { a } 12 \\
\text { cigarrillos, De } 4 \text { a } 6 \text { cigarrillos, De } 1 \text { a } 3 \\
\text { cigarrillos, Ninguna }\end{array}$ & 11 & $\begin{array}{l}\text { Usted fuma si está: } \\
\text { Solo, con amigos, entre familia, con mi } \\
\text { pareja , ninguno }\end{array}$ \\
\hline 4 & $\begin{array}{l}\text { ¿Cree Ud. ¿Que los fumadores consumen } \\
\text { cigarrillos por alguna de estas causas? } \\
\text { Ansiedad, perdidas afectivas, tristeza, } \\
\text { estrés }\end{array}$ & 12 & $\begin{array}{l}\text { ¿Recurre al cigarrillo para ...? } \\
\text { Sentirse bien físicamente, Despejar su } \\
\text { mente, ambos, ninguno }\end{array}$ \\
\hline 5 & $\begin{array}{l}\text { ¿si usted considera que un fumador puede } \\
\text { influenciado por alguno de estos factores? } \\
\text { Amigos, pareja, colegas, familiares }\end{array}$ & 13 & $\begin{array}{l}\text { ¿Cree que fumar le ayuda a reducir el } \\
\text { apetito y en parte le ayuda a bajar de peso? } \\
\mathrm{Si}, \mathrm{No}\end{array}$ \\
\hline
\end{tabular}




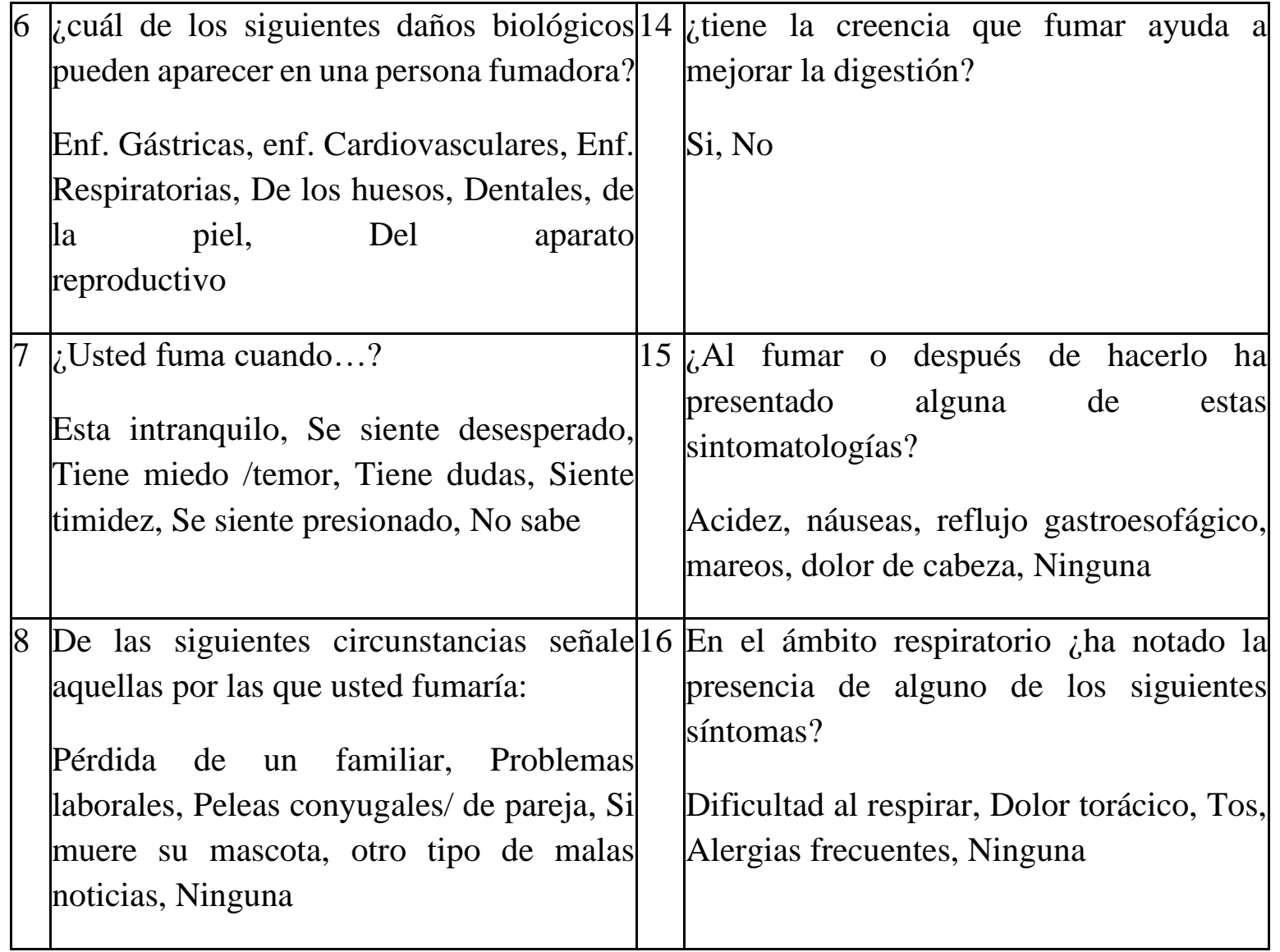

Todos estos ítems fueron debidamente verificados y aprobados mediante una rúbrica de confiabilidad en la cual se determinó los siguientes parámetros: VALIDEZ, PERTINENCIA y COHERENCIA del test; a los cuales se les otorgó una calificación de 0 a 100 en cada uno de los ítems que conforman el cuestionario. Posteriormente cada valor fue sumado y promediado acorde con cada uno de los parámetros establecidos dando como resultado el puntaje siguiente: para el parámetro de Validez se obtuvo un total de 97, 68 puntos, en Pertinencia un valor de 97,75 puntos y en Coherencia un valor de 97, 62 puntos. Los tres puntajes totales se promediaron entre sí para dar un total de 97,68 que, acorde con la rúbrica de confiabilidad se determinó que el instrumento tiene la calidad de MUY CONFIABLE, y fue aprobado para su ejecución en fase piloto.

La población es finita y está conformada por 153 estudiantes de $8^{\circ}$ semestre correspondientes a las carreras de Ing. Industrial e Ing. En sistemas de la Universidad Estatal de Milagro, legalmente matriculados, pertenecientes la jornada académica nocturna. La muestra es no probabilística. Criterios de selección de individuos: estudiantes matriculados en octavo ciclo en las carreras de ingeniería en sistemas e ingeniería industrial de la sección nocturna. Estos criterios no pudieron ser cumplidos por las otras carreras que conforman la facultad (biotecnologías) debido a que no poseen octavo ciclo por ser de reciente apertura. 


\section{Resultados.}

Los resultados fueron procesados mediante el programa ®IBM SPSS Statistics 21, en la cual se insertó las variables de la encuesta, se les asignó una etiqueta, y se determinó los valores en escala, los nominales y los ordinales, así como el tipo de variable a la que pertenecen. Y se muestran en la siguientes tablas y gráficos los porcentajes con cada uno de los indicadores aplicados a la población asociados a la edad y el género de la población.

Tabla 2. Hábito de fumar

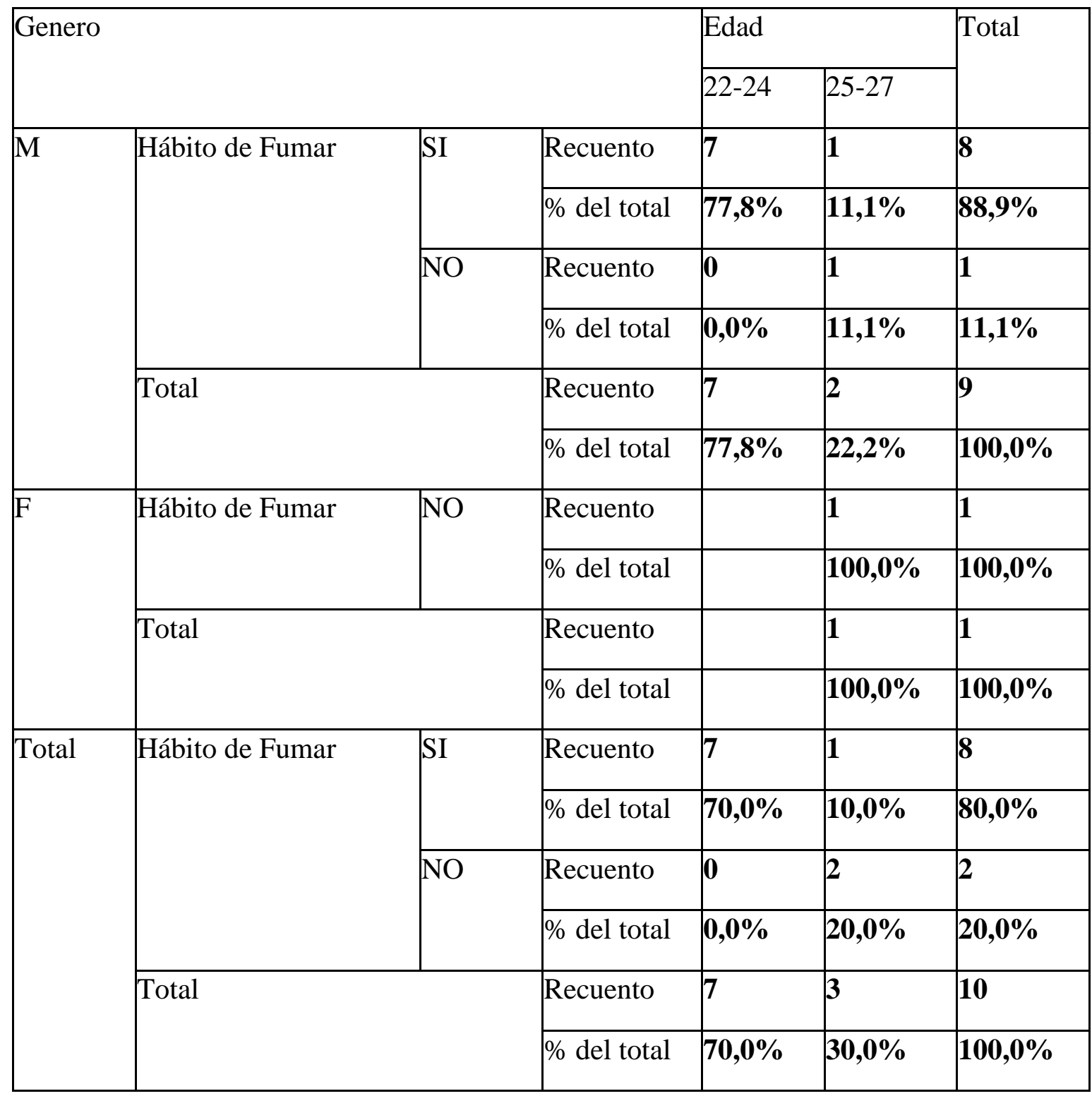

La tabla 2 mostró que en cuanto al hábito de fumar existe un 77,8\% de población masculina con edades entre 22 a 24 años que afirman ser fumadores, y un 11,1\% que corresponde a edades entre 25 a 27 años también aseguran ser parte de este hábito. Por otra parte, la población femenina negó estar asociada con este tipo de adicciones. 
Tabla 3. Frecuencia de consume

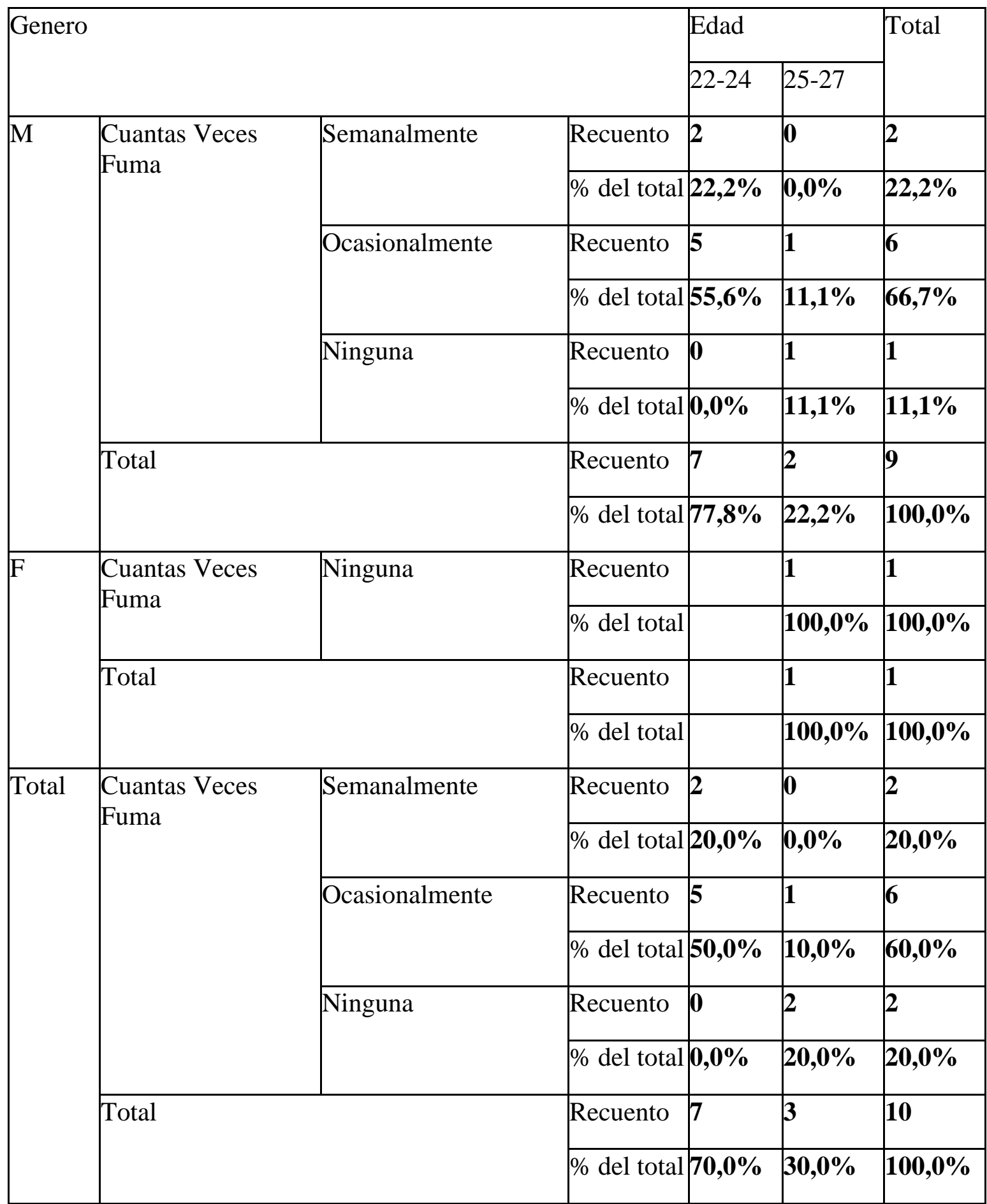

En lo que concierne a la frecuencia con que los estudiantes consumen tabaco la tabla 2 mostró que de la población masculina con edades comprendidas entre 22 a 24 años un 55,6\% afirmó 
que fuman ocasionalmente, un 22,2\% de jóvenes con el mismo rango de edad reveló que lo hace de manera semanal. Por otra parte, la población femenina afirmó que no fuma.

\section{Gráfico 1. Cantidad de consumo}

El grafico 1 demuestra que de la población masculina comprendida en edades de 22 a 24 años el 66,67\% consume de 1 a 3 cigarrillos cuando fuma, el 11,11\% consume de 4 a 6 cigarrillos, por otra parte, la población femenina no se pronunció respecto al consumo de tabaco.

Tabla 4. Situaciones que generan ansiedad

\begin{tabular}{|c|c|c|c|c|c|c|}
\hline \multicolumn{4}{|c|}{ GENERO } & \multicolumn{2}{|l|}{ EDAD } & \multirow[t]{2}{*}{ Total } \\
\hline & & & & $22-24$ & $25-27$ & \\
\hline \multirow[t]{6}{*}{$M$} & \multirow{4}{*}{$\begin{array}{l}\text { Situaciones que } \\
\text { Generan Ansiedad }\end{array}$} & \multirow[t]{2}{*}{ Esta Intranquilo } & Recuento & 4 & 1 & 5 \\
\hline & & & $\%$ del total & $44,4 \%$ & $11,1 \%$ & $55,6 \%$ \\
\hline & & \multirow[t]{2}{*}{ No Sabe } & Recuento & 3 & 1 & 4 \\
\hline & & & $\%$ del total & $33,3 \%$ & $11,1 \%$ & $44,4 \%$ \\
\hline & \multirow{2}{*}{\multicolumn{2}{|c|}{ Total }} & Recuento & 7 & 2 & 9 \\
\hline & & & $\%$ del total & $77,8 \%$ & $22,2 \%$ & $100,0 \%$ \\
\hline \multirow[t]{4}{*}{$\mathrm{F}$} & \multirow{2}{*}{$\begin{array}{l}\text { Situaciones Que } \\
\text { Generan Ansiedad }\end{array}$} & \multirow[t]{2}{*}{ No Sabe } & Recuento & & 1 & 1 \\
\hline & & & $\%$ del total & & $100,0 \%$ & $100,0 \%$ \\
\hline & \multirow{2}{*}{\multicolumn{2}{|c|}{ Total }} & Recuento & & 1 & 1 \\
\hline & & & $\%$ del total & & $100,0 \%$ & $100,0 \%$ \\
\hline \multirow[t]{6}{*}{ Total } & \multirow{4}{*}{$\begin{array}{l}\text { Situaciones Que } \\
\text { Generan Ansiedad }\end{array}$} & \multirow[t]{2}{*}{ Esta Intranquilo } & Recuento & 4 & 1 & 5 \\
\hline & & & $\%$ del total & $40,0 \%$ & $10,0 \%$ & $50,0 \%$ \\
\hline & & \multirow[t]{2}{*}{ No Sabe } & Recuento & 3 & 2 & 5 \\
\hline & & & $\%$ del total & $30,0 \%$ & $20,0 \%$ & $50,0 \%$ \\
\hline & \multirow{2}{*}{\multicolumn{2}{|c|}{ Total }} & Recuento & 7 & 3 & 10 \\
\hline & & & $\%$ del total & $70,0 \%$ & $30,0 \%$ & $100,0 \%$ \\
\hline
\end{tabular}

La tabla 3 determinó el porcentaje de situaciones en las que los jóvenes son propensos a sufrir ansiedad, y en la cuales recurren al consumo de tabaco. El 44,4\% de los jóvenes de género 
masculino con edades entre 22 a 24 años consume cigarrillos cuando sienten intranquilidad, $11,1 \%$ entre los 25 y 27 años también lo hacen por esta misma razón. El 44,4\% restante afirma no saber que situaciones los inducen a fumar.

\section{Gráfico 2. Situaciones que generan depresión}

El grafico 2 muestra las situaciones depresivas en las cuales los jóvenes pueden ser influenciados a fumar. El 33,3\% de jóvenes con edades entre los 22 y 24 años aseguró que consumen tabaco cuando reciben malas noticias, lo cual genera un malestar en ellos, el 11,1\% lo hace cuando sienten mucha tristeza, como en casos de pérdidas de familiares. El otro $33,3 \%$ no sabe que situaciones pueden conducirlos a la depresión y por lo tanto a consumir tabaco.

\section{Gráfico 3. Influencia social en el consumo de tabaco}

En el gráfico 3 muestra que la influencia social está presente en los jóvenes universitarios y pueden conducirlos a adquirir conductas tabáquicas. De esta manera el 55,56\% de jóvenes con edades entre 22 y 24 años afirma fumar en compañía de sus amistades. Y el 11,1\% entre los 25 y 27 años también corrobora este hecho. El 22,22\% restante dice no hacerlo con ninguna de las opciones mencionadas en la encuesta. (familia, colegas).

Los resultados presentados revelan que un porcentaje importante de los estudiantes universitarios de género masculino de las carreras de ingeniería $(77,8 \%)$ son fumadores y así lo confirmaron en la encuesta realizada. Acorde con diversos estudios se ha estimado que, en todo el mundo, el 29\% de la población de 15 años en adelante, posee las tasas globales más altas en Europa y Asia Central (35\%). A nivel mundial, los hombres fuman más que las mujeres (47\% vs $11 \%$, respectivamente), y la prevalencia de picos diarios de tabaquismo en edades de 30-49 años es de 36-37\% (Bardach, A. 2016). Aunque varía por país, la prevalencia de tabaquismo en adultos en América Latina que se ha reportado es de aproximadamente $40 \%$ para los hombres y $24 \%$ para las mujeres, con una prevalencia global del 32\%. Un informe de la OMS de 2008 publicó estimaciones de prevalencia ajustadas que son inferiores a las reportadas en años anteriores. Además, se confirmó que de la población fumadora el 55,6\% son fumadores ocasionales y el 22\% lo hacen semanalmente, además de consumir un considerable número de cigarrillos (66,67\% entre 1 a 3 cigarrillos). Estos datos revelan que los estudiantes universitarios que consumen tabaco de manera frecuente, pueden estar sometidos a la presencia de diversos factores, los cuales se originan principalmente en su lugar de estudios que es donde pasan la mayor parte de su tiempo y donde se relacionan e interactúan más con otras personas. En base a esta información se incluyó en el test algunas preguntas que determinaron la asociación de factores psicológicos y sociales con el consumo de tabaco. Tal como se lo determinó en el siguiente estudio la experimentación de tabaco se asoció con la presencia de sentimientos de soledad y bajo número de amigos; esta asociación se mantuvo con el uso frecuente de tabaco. Para lo cual se encontró en los estudiantes 
universitarios que el 44,4\% recurre al tabaco cuando siente intranquilidad, lo cual puede estar ligado a bajos niveles de ansiedad; y el 33,3\% lo hace cuando reciben malas noticias o presentan situaciones depresivas (WHO, 2008).

Actualmente, se reconoce que la dependencia al tabaco es un fenómeno conductual multidimensional que involucra componentes psicológicos y sociales, además de físicos. Adicionalmente la evidencia independiente y la propia documentación de la industria tabacalera dejan claro que las compañías tabacaleras han utilizado las características de diseño y aditivos químicos en el proceso de fabricación, de manera que aumente el impacto de la nicotina, el agente adictivo en los productos del tabaco. Algunas de las formas en que el poder adictivo de los cigarrillos se ha desarrollado incluyen: aumento de los niveles de nicotina, adición de amoniaco o compuestos de amoniaco que aumentan la velocidad a la que la nicotina se libera en el cerebro, adición de azúcares, que aumentan los efectos adictivos de la nicotina y facilitan la inhalación del humo del tabaco (Burns, D. 2014). Además de vigilar las propiedades adictivas de sus productos, las empresas de tabaco también manejan su publicidad de manera que agraden a nuevos fumadores y desarrollan la probabilidad de que se conviertan en consumidores usuales.

Por otra parte, la influencia social está presente en los jóvenes universitarios y pueden conducirlos a adquirir conductas tabáquicas. De acuerdo a los resultados el 55,56\% de jóvenes con edades entre 22 y 24 años afirma fumar en compañía de sus amistades. Se dice que tener amigos fumadores se asocia fuertemente con ser fumador, según estudios realizados en España, muy posiblemente por las relaciones que establecen durante su etapa formativa y académica. Algunos estudiantes utilizan el tabaquismo como un mecanismo de supervivencia, jóvenes de 18 a 24 años de edad prueban el cigarro por primera vez en la universidad. Los estudiantes que en la secundaria fumaban, tienen más probabilidades de aumentar la frecuencia y la cantidad de consumo de tabaco en la universidad. En diversos estudios se han identificado características asociadas al consumo: estudiantes del sexo masculino, amigos fumadores, estrés, semestres avanzados de la carrera, tener una imagen social deseada, o como facilitador para establecer relaciones interpersonales; son algunos factores que se han reportado según estudios realizados en México (Ortega-Ceballos, P. 2018). Por lo cual se los ha determinado como factores principales dentro de la relación con el consumo de tabaco.

\section{Conclusiones.}

- Se ha determinado que la nicotina es una sustancia altamente adictiva. Si los jóvenes la consumen antes de los 25 años, puede causarles adicción y alterar el desarrollo de su sistema nervioso y respiratorio. Además, al alterarse el sabor, el olor y otros atributos sensoriales de sus productos, los fabricantes de tabaco facilitan que comiencen y continúen fumando los nuevos usuarios, la gran mayoría de los cuales son jóvenes. 
- El campus Universitario es un ambiente educacional de igual importancia que un lugar de trabajo, donde los estudiantes, facultades, y personal permanece muchas horas al día. En base a esto, el campus se constituye en un ambiente importante donde se trata de proteger la salud de los estudiantes y empleados siguiendo un modelo de comportamientos saludables.

- Los resultados de este análisis de trabajo de campo sugieren que factores de tipo psicológico y sociales influyen ampliamente en el inicio del consumo de tabaco, cabe recalcar que la validación de este instrumento establece un rango de confiabilidad aceptable para su ejecución. Por lo tanto, es necesario establecer una propuesta adecuada para esta problemática, principalmente porque los principales afectados son los jóvenes en etapa estudiantil quienes a su vez pueden inducir a otros a iniciarse tempranamente en esta práctica que cada vez va en aumento dentro de la población juvenil.

\section{Referencias Bibliográficas.}

Carrión, D., César, P., Calle, S., \& Silvana, L. (2017). “Ansiedad y consumo de tabaco en los estudiantes de la Carrera de Psicología Clínica, de la universidad nacional de Chimborazo, periodo abril-septiembre 2016" (Bachelor's thesis, Universidad Nacional de Chimborazo, 2017).

Armijos, Ordóñez, L. E. (2017). Consumo de alcohol y tabaco y relación con la estructura y funcionalidad familiar en estudiantes del Bachillerato del Instituto Tecnológico Nuestra Señora del Rosario de Catamayo en el periodo 2016-2017 (Bachelor's thesis).

Zander Neves, C., Devicari Bueno, C., Pires Felden, G., Costa Irigaray, M., Rivadeneira, M. F., Soares Xavier Oenning, N., \& Goulart, B. N. (2018). Tabaco en adolescentes escolares brasileños: asociación con salud mental y contexto familiar. Gaceta Sanitaria, 32, 216-222.

Monteiro, M. G. (2007). Alcohol y Salud Pública en las Américas: un caso para la acción. Organización Panamericana de la Salud.

Martínez, J. C. (2016). Factores asociados a la mortalidad por enfermedades no transmisibles en Colombia, 2008-2012. Biomédica, 36(4), 535-546.

Wünsch Filho, V., Mirra, A. P., López, R. V. M., \& Antunes, L. F. (2010). Tabagismo e câncer no Brasil: evidências e perspectivas. Revista Brasileira de Epidemiologia, 13, $175-187$

Danel, I. (2018). Intercambio de conocimientos para mejorar la salud en la Región de las Américas. American Journal of Public Health, 108(Suppl 6), S412. 
Organización Mundial de la Salud, Informe sobre la epidemia mundial del tabaquismo 2017, https://apps.who.int/iris/bitstream/handle/10665/258599/WHO-NMH-PND-17.4spa.pdf;jsessionid=3684A56B442357EDAE43237874F26DBE?sequence $=1$

Paz-Ballesteros, W. C., Zavala-Arciniega, L., Gutiérrez-Torres, D. S., Ponciano-Rodríguez, G., \& Reynales-Shigematsu, L. M. (2019). Evaluación de la dependencia física y psicológica al tabaco en fumadores mexicanos adultos, Encodat 2016. salud pública de méxico, 61(2, Mar-Abr), 136-146

Castedo, C. R., de Granda-Orive, J. I., \& González-Barcala, F. J. (2019). Incremento de la prevalencia del tabaquismo: ¿causas y actuación? Archivos de Bronconeumología.

Cecilia, M. J., Atucha, N. M., \& García-Estañ, J. (2017). Estilos de salud y hábitos saludables en estudiantes del Grado en Farmacia. Educación Médica.

Bello, S., Flores, A., Bello, M., \& Chamorro, H. (2009). Diagnóstico y tratamiento psicosocial del tabaquismo. Revista chilena de enfermedades respiratorias, 25(4), 218-230.

Mandujano, J. L., Piña, E. V., Lauriano, M. N., Puebla, D. P., Chainé, S. M., \& Morales, S. E. C. (2018). Ansiedad, depresión, afecto negativo y positivo asociados con el consumo de tabaco en universitarias. Revista Internacional de Investigación en Adicciones, 4(1), 4-12.

Hernández Sampieri, R. Metodología de la investigacion (Sexta ed.). 2016. Mexico: McGraw Hill interamericana.

Bardach, A., Perdomo, H. A. G., Gándara, R. A. R., \& Ciapponi, A. (2016). Niveles de ingreso y prevalencia de tabaquismo en América Latina: revisión sistemática y metaanálisis. Revista Panamericana de Salud Pública, 40, 263-2

Champagne, B. M., Sebrié, E. M., Schargrodsky, H., Pramparo, P., Boissonnet, C., \& Wilson, E. (2010). Tobacco smoking in seven Latin American cities: the CARMELA study. Tobacco control, 19(6), 457-462.

Jha, P., Jha, P., Chaloupka, F. J., Jha, P., Chaloupka, F. J., Corrao, M., ... \& Jha, P. (2006). Reducing the burden of smoking world-wide: effectiveness of interventions and their coverage. Drug and alcohol review, 25(6), 597-609.

World Health Organization, \& Research for International Tobacco Control. (2008). WHO report on the global tobacco epidemic, 2008: the MPOWER package. World Health Organization.

Guindon, G. E., \& Boisclair, D. (2003). Past, current and future trends in tobacco use. 
Elias, J., Hendlin, Y. H., \& Ling, P. M. (2018). Public versus internal conceptions of addiction: An analysis of internal Philip Morris documents. PLoS medicine, 15(5), e1002562.

Paz-Ballesteros, W. C., Zavala-Arciniega, L., Gutiérrez-Torres, D. S., Ponciano-Rodríguez, G., \& Reynales-Shigematsu, L. M. (2019). Evaluación de la dependencia física y psicológica al tabaco en fumadores mexicanos adultos, Encodat 2016. salud pública de méxico, 61(2, Mar-Abr), 136-146.

Huang, H. W., Lu, C. C., Yang, Y. H., \& Huang, C. L. (2014). Comportamientos del tabaquismo en adolescentes, influidos por el tabaquismo de profesores, familiares y amigos. International nursing review en español: revista oficial del Consejo Internacional de Enfermeras, 61(2), 238-245.

Ortega-Ceballos, P. A., Terrazas-Meraz, M. A., Arizmendi-Jaime, E. R., \& TapiaDomínguez, M. (2018). Conocimientos, actitudes y factores asociados al consumo de tabaco en estudiantes universitarios de enfermería. Enfermería universitaria, 15(2), $159-171$.

Burns, D., Donny, E., Hatsukami, D., Hecht S., Henningfield, J. (2014). Designed for addiction. United States. 6-10. 


\section{PARA CITAR EL ARTÍCULO INDEXADO.}

Gavino Díaz, G. E., López Rivadeneira, E. J., Guaraca Parreño, V. H., \& Romero Urrea, H. E. (2019). Factores que inciden en el consumo de tabaco en los estudiantes de ingeniería: estudio piloto. Ciencia Digital, 3(4), 55-70. https://doi.org/10.33262/cienciadigital.v3i4.910

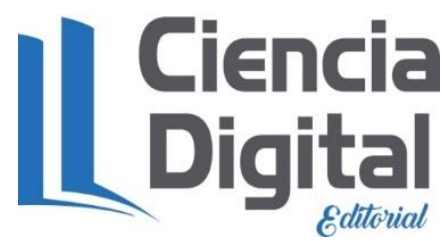

El artículo que se publica es de exclusiva responsabilidad de los autores y no necesariamente reflejan el pensamiento de la Revista Ciencia Digital.

El artículo queda en propiedad de la revista y, por tanto, su publicación parcial y/o total en otro medio tiene que ser autorizado por el director de la Revista Ciencia Digital.
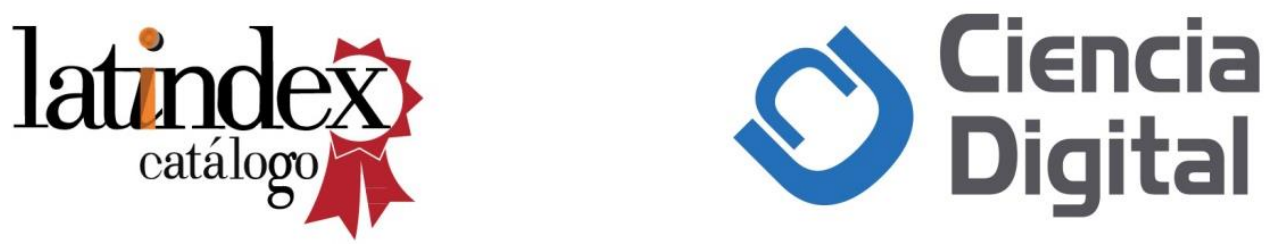